\title{
Determination of HIV-1 coreceptor tropism using proviral DNA in women before and after viral suppression
}

\author{
Russell E Baumann ${ }^{1}$, Amy A Rogers ${ }^{1}$, Hasnah B Hamdan ${ }^{1}$, Harold Burger ${ }^{2}$, Barbara Weiser ${ }^{2}$, Wei Gao ${ }^{3}$, \\ Kathryn Anastos ${ }^{3}$, Mary Young ${ }^{4}$, William A Meyer $1 I^{5}$, Rick L Pesano ${ }^{1}$ and Ron M Kagan ${ }^{{ }^{*}}$
}

\begin{abstract}
Background: An HIV-1 tropism test is recommended prior to CCR5 antagonist administration to exclude patients harboring non-R5 virus from treatment with this class of antiretrovirals. HIV-1 tropism determination based on proviral DNA (pvDNA) may be useful in individuals with plasma viral RNA suppression. We developed a genotypic tropism assay for pvDNA and assessed its performance in a retrospective analysis of samples collected longitudinally.

Results: We randomly selected paired plasma/PBMC samples from the Women's Interagency HIV Study with plasma viral load $\geq 5,000 \mathrm{cp} / \mathrm{mL}$ at time 1 (T1), undetectable viral load maintained for $\geq 1$ year and CD4+ >200 cells/ $\mathrm{LL}$ at time 2 (T2). pvDNA was isolated from cryopreserved PBMCs. Sequences were analyzed in triplicate from 49/50 women, with tropism assigned using the geno2pheno (g2p) algorithm. The median time between T1 and T2 was 4.1 years. CXCR4-using virus was detected in 24\% of the RNA samples and 33\% of the pvDNA samples at T1, compared to $37 \%$ of the pvDNA samples at T2. Concordance between plasma RNA and pvDNA tropism was 88\% at T1 and 80\% at T2. The g2p scores for RNA (T1) vs DNA $(T 1, T 2)$ were strongly correlated (Spearman rho: 0.85 (T1); 0.78 (T2)). In women with evidence of tropism switch at T2 (either R5 to non-R5 or non-R5 to R5), there was a correlation between change in tropism and time. Mean pvDNA viral load decreased by $0.4 \log 10$ copies/106 cells between T1 and T2 ( $p<0.0001)$, but this decrease was not significantly associated with tropism status.

Conclusions: We demonstrated that pvDNA tropism in women with HIV-1 suppression is concordant with prior RNA tropism results, even after a median time of $>4$ years. pvDNA tropism testing may be useful to determine eligibility of patients with viral suppression to switch to a CCR5-antagonist based regimen as well as for research purposes.
\end{abstract}

Keywords: HIV, Tropism, pvDNA

\section{Background}

Human immunodeficiency virus type 1 (HIV-1) infects cells through interaction with the CD4 receptor and one of two coreceptors, CCR5 or CXCR4 [1-3]. CCR5 coreceptor-using virus (R5 virus) predominates in $80-90 \%$ of recently infected and treatment-naïve HIV-1 patients, while mixed populations of R5 virus and CXCR4 coreceptor-using virus (non-R5 or "X4" virus) are found in up to $50 \%$ of late-stage and antiretroviral treatment (ART)-experienced patients [4-10]. The presence of

\footnotetext{
* Correspondence: Ron.M.Kagan@questdiagnostics.com

${ }^{1}$ Focus Diagnostics, a Subsidary of Quest Diagnostics, San Juan Capistrano, CA, USA

Full list of author information is available at the end of the article
}

non-R5 virus is associated with lower CD4+ T-cell counts, higher plasma viral loads, and more rapid progression to AIDS $[6,9,11,12]$. Small-molecule CCR5 antagonists such as maraviroc can effectively inhibit the interaction of R5 HIV-1 with the CCR5 coreceptor $[3,13]$. An HIV-1 coreceptor tropism test is required prior to maraviroc administration, to exclude patients harboring non-R5 virus from treatment with this drug (Maraviroc prescribing information, https://www.gsksource. com/gskprm/htdocs/documents/SELZENTRY-PI-MG.PDF).

Many HIV-1-infected individuals undergoing ART achieve undetectable or low levels plasma HIV-1 RNA. Because most plasma RNA tropism tests require at least 1,000 copies/mL of HIV-1 RNA to be present in order to 
obtain a reportable result, a different type of assay would be required to determine whether individuals with viral suppression could benefit from the inclusion of a CCR5 antagonist in their ART regimen. In this situation, tropism testing of archived HIV-1 proviral DNA (pvDNA) in peripheral blood mononuclear cells (PBMC) can be performed $[14,15]$. From a clinical standpoint, the ability to determine tropism from pvDNA allows testing to be extended to persons who are taking effective antiretroviral therapy but who may be candidates for CCR 5 antagonist therapy. This includes patients who are experiencing adverse effects from their current regimen and patients receiving complex regimens who may benefit from regimen simplification. A number of studies have shown strong agreement between plasma RNA and pvDNA tropism [16-20]. However, few studies have examined pvDNA tropism longitudinally in individuals taking suppressive ART [21-23], and most previous studies of HIV-1 tropism have focused mostly on men $[4,8,12,16]$.

We have developed a genotypic tropism assay that uses triplicate PCR amplification of the HIV-1 envelope V3 region, the major determinant of coreceptor tropism $[1,3]$, and Sanger sequencing of pvDNA isolated from PBMCs [16]. Here we assessed its performance in a retrospective longitudinal analysis of samples from individuals in the Women's Interagency HIV Study (WIHS), an ongoing long-term observational cohort study of 3,772 HIV-1 infected or at- risk women [24].

\section{Results}

\section{Baseline characteristics}

HIV-1 envelope V3 loop sequences from plasma RNA and pvDNA at T1 and pvDNA at T2 were successfully obtained from 49/50 HIV-positive women and 0/10 HIVnegative control samples. The median interval between time point T1 and T2 samples was 4.1 years (IQR: 2.6, 5.1 years). Baseline characteristics of the subjects are shown in Table 1 . There was no statistically significant association between age, viral load at time point $\mathrm{T} 1$, nadir CD4+ T-cell count, T-cell counts at either time point or the interval between time points and the tropism concordance status between pvDNA and plasma RNA tropism tests (Table 1). Women with concordant non-R5 pvDNA and plasma RNA tropism results appeared to have a lower nadir CD4+ count (138 cell/uL vs 194 cells/uL overall) but this difference was not statistically significant.

\section{Coreceptor tropism concordance between plasma RNA and pvDNA}

V3 sequences were classified as R5 for 57\% (28/49) and non-R5 for 20\% (10/49) of the women for analyses of both RNA (T1) and pvDNA (T1 and T2). Tropism results were discordant for 11 women (Figure 1). Four women had only R5 virus detectable in the plasma RNA sample and pvDNA sample collected at T1 but detectable non-R5 virus in the later pvDNA sample from T2 (subjects 1, 2, 5 and 9). An additional four women had only R5 virus in the plasma RNA sample collected at T1 and detectable non-R5 virus for both pvDNA time points (subjects 3, 4, $7,10)$. Two women had detectable non-R5 virus in both the plasma RNA sample and the pvDNA sample at T1 but only $\mathrm{R} 5$ virus was detected in the pvDNA sample at time point T2 (6 and 11). The last discordant sample had only detectable R5 virus in the plasma RNA sample at time point T1, non-R5 virus in the T1 pvDNA sample, but only R5 virus in the T2 pvDNA sample (subject 8 ). Phylogenetic tree analysis of the 11 discordant samples showed that the T2 pvDNA sequences remained clustered with sequences from $\mathrm{T} 1$ from the same subject, illustrating that they were closely related. The discordant non-R5 viruses tended to appear on distinct branchings within each cluster (Figure 1) showing that they were distinct yet related phylogenetically. None of the discordant sequences clustered with sequences from an unrelated patient indicating

Table 1 Subject Demographic, Virologic and Immunologic Parameters

\begin{tabular}{|c|c|c|c|c|c|}
\hline \multirow[b]{2}{*}{ Median (IQR) ${ }^{1}$} & \multirow[b]{2}{*}{ All Subjects } & \multicolumn{3}{|c|}{ Coreceptor Tropism Status ${ }^{2}$} & \multirow[b]{2}{*}{$p$-value ${ }^{3}$} \\
\hline & & Concordant R5 & Concordantnon-R5 & Discordant & \\
\hline $\mathrm{N}$ & 49 & 28 & 10 & 11 & \\
\hline Age (years) & $38(33,43)$ & $39(32,33)$ & $36(32,38)$ & $40(34,44)$ & 0.62 \\
\hline Nadir CD4+(cell/uL) & $191(87,269)$ & $194(106,270)$ & $138(44,249)$ & $191(83,285)$ & 0.58 \\
\hline T1 CD4+(cells/uL) & $323(243,417)$ & $321(235,404)$ & $276(234,375)$ & $345(276,537)$ & 0.70 \\
\hline T1 viral load(log $\log _{10}$ copies $\left./ \mathrm{mL}\right)$ & $4.8(4.4,5.3)$ & $4.7(4.2,5.3)$ & $5.1(4.5,5.4)$ & $4.8(4.4,5.3)$ & 0.41 \\
\hline Delta T1 and T2 (years) & $4.1(2.6,5.1)$ & $3.1(2.3,4.7)$ & $4.2(2.7,5.4)$ & $4.3(3.3,6.2)$ & 0.28 \\
\hline T2 CD4+(cells/uL) & $558(365,717)$ & $576(258,747)$ & $478(294,772)$ & $532(371,666)$ & 0.81 \\
\hline
\end{tabular}

${ }^{1} \mathrm{~T} 1$ : Time point 1, viremic subjects pre-suppression; T2: Time point 2 , after subjects had undetectable viral load $(<80 \mathrm{copies} / \mathrm{mL})$ for $\geq 1$ year.

${ }^{2}$ Concordant R5: CCR5-tropic viral RNA at T1 and proviral DNA at T1 and T2; Concordant non-R5: CXCR4-tropic viral RNA at T1 and proviral DNA at T1 and T2; Discordant: at least 1 discordant tropism result at $\mathrm{T} 1$ or $\mathrm{T} 2$.

${ }^{3}$ Kruskall-Wallis test for difference in distributions among tropism status categories. 


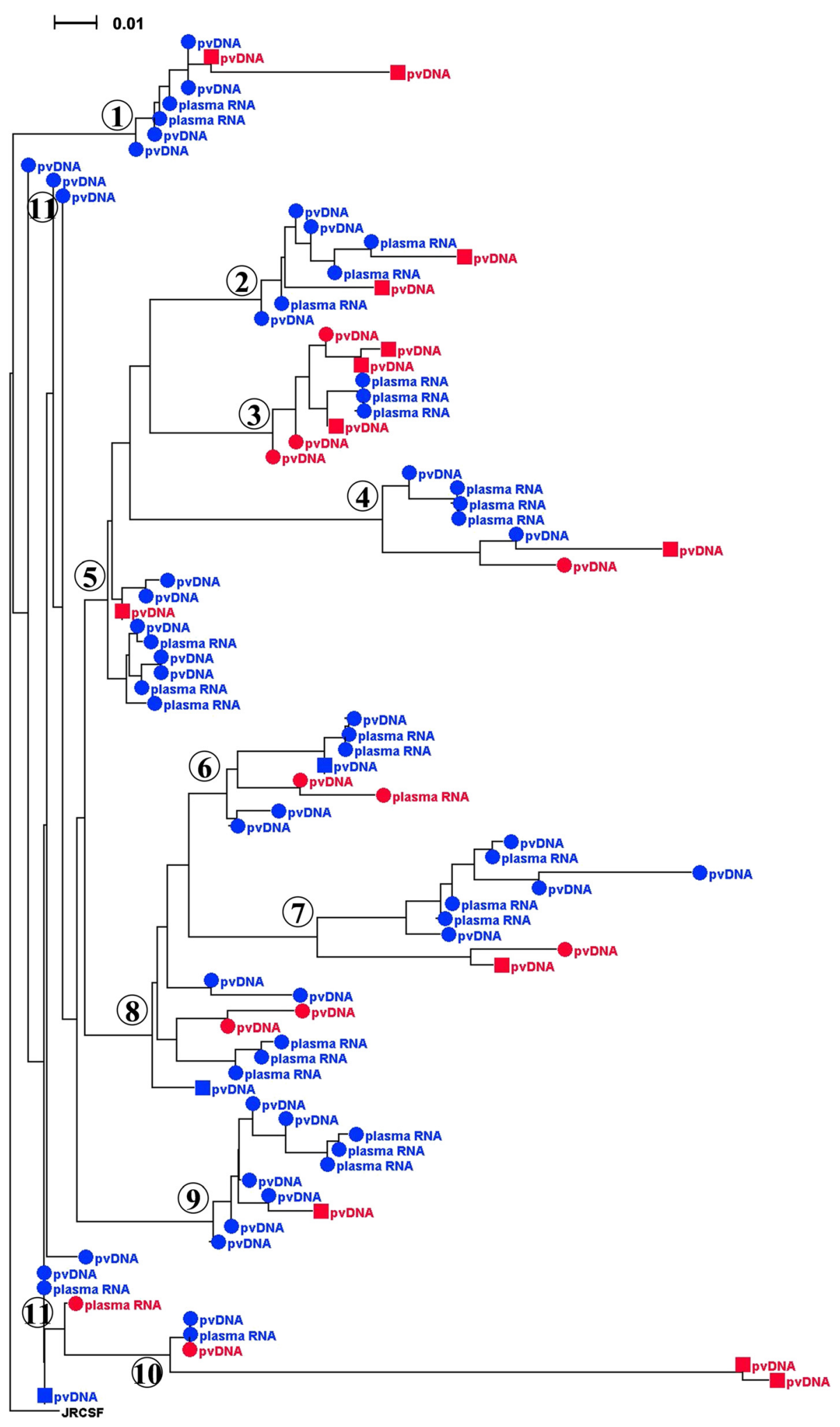

Figure 1 Phylogeny of V3 sequences for discordant tropism group. Blue circles: T1 R5. Red circles: T1 non-R5. Blue squares: TP2 R5. Red squares: T2 non-R5. JRCSF: V3 loop of R5 reference sequence used as an outgroup. Subjects are consecutively numbered $1-11$. The scale bar is shown in the upper left corner. V3 sequences were aligned with ClustalX version 2.1 (http://www.clustal.org) and a neighbor-joining tree was generated using Kimura-corrected distances for multiple substitutions. The tree was then rendered and illustrated with Denodroscope version 3.2.10 (http://ab.inf.uni-tuebingen.de/software/dendroscope/). 
that inter-sample contamination is unlikely to account for the observed discordances.

A higher proportion of pvDNA samples were determined to have non- $\mathrm{R} 5$ tropism (33\% at $\mathrm{T} 1$ and $37 \%$ at $\mathrm{T} 2$ ) as compared to plasma RNA ( $24 \%$ at T1). The overall concordance of tropism predictions between viral RNA and pvDNA, however, was $88 \%$ (kappa $=0.70)$ at $\mathrm{T} 1$; concordance was $80 \%(\mathrm{kappa}=0.53)$ at T2 (Table 2). pvDNA was 92\% sensitive for non-R5 virus relative to plasma RNA at T1 but the sensitivity was lower at T2 (83\%) after a median interval of 4.1 years (Table 2). The geno2pheno FPR scores for coreceptor tropism prediction were highly correlated between RNA and pvDNA at T1 (Spearman correlation coefficient rho $=0.85)$ and $\mathrm{T} 2($ rho $=0.78)$, and between the pvDNA results at $\mathrm{T} 1$ and $\mathrm{T} 2(\mathrm{rho}=0.85)$.

Lymphocyte counts and pvDNA copy number at T1 and T2 Although CD4+ cell counts did not differ significantly as a function of tropism status, women with non-R5 virus tended to have a lower CD4+ count (Table 1). Women who had only R5 virus at both time points had the largest gain in CD $4+$ cells $(237$ cells $/ \mu \mathrm{L}$ ) over that time period, whereas women harboring non-R5 virus at both time points had a smaller CD4+ cell count increase between T1 and T2 (104 cells/ $\mu \mathrm{L})$. The women with discordant tropism results (defined as having a tropism discordance between either PBMC time point or plasma at T1 and one of the PBMC timepoints), had a median increase of 169 cells $/ \mu$ L. For women with any non-R5 result at either time point, the median increase was 159 cells/ $\mu \mathrm{L}$ (IQR: $-4.0,+321 ; \mathrm{p}=0.049)$. The difference in CD4+ change between the R5 and non-R5 groups was not statistically significant ( $\mathrm{p}=0.13$; Additional file 1: Figure S1).

pvDNA copy number was also measured at both time points. The mean pvDNA viral load decreased from $3.2 \log _{10}$ copies $/ 10^{6}$ cells at T1 to $2.8 \log _{10}$ copies $/ 10^{6}$ cells at T2, a significant decline of $0.4 \log _{10}$ copies $/ 10^{6}$ cells $(\mathrm{p}<0.0001$; Additional file 2: Figure S2). Changes in proviral DNA viral load were not significantly associated with baseline tropism status.

\section{Discussion}

Investigation of viral evolution during suppressive ART has not documented viral replication in an examination of plasma [25]. Our study compared proviral V3 sequences from PBMCs before and years after viral suppression. There was no evidence of a tropism switch for 38 women with HIV-1 suppression over a median of over 4 years. This observation is consistent with low rates of viral evolution of the V3 loop. Five women with viral suppression had evidence of a tropism switch defined as discordance between the pvDNA tropism results at the two time points, irrespective of the RNA tropism at T1. A potential explanation for this phenomenon could be low level viral replication that was below the limit of quantitation ( 80 copies $/ \mathrm{mL}$ ) of the plasma viral load assays used in the WIHS. It is also consistent with some viral replication persisting in cells during viral suppression in plasma $[21,26]$. Clonal expansion of latently infected cells during suppressive ART has also been described [27]. Persistence of expanded clones is often associated with viral integration in genes controlling cell growth. This phenomenon could potentially alter the proportion of non-R5 virus over prolonged periods of virologic suppression. Two other studies that investigated tropism evolution in suppressed patients found lower rates of tropism switching of $7.1 \%(\mathrm{~N}=128$, median suppression 4 years) [28] and $9.5 \%$ respectively $(\mathrm{N}=42$, median suppression 2 years) [21]. These studies however, were performed on more recently supressed patients, used a viral load cutoff of 50 copies/mL and included both male and female subjects.

The sensitivity of pvDNA tropism determination at T2 with respect to plasma RNA after a median of 4.1 years of virologic suppression was lower compared to that of the pre-suppression sample T1. Next Generation Sequencing (NGS) of HIV-1 plasma RNA has been shown to predict virologic response to maraviroc as well as the enhanced sensitivity Trofile phenotypic assay and with greater sensitivity for minority non-R5 variants than population sequencing [29-31]. However, the accuracy of NGS for tropism prediction using pvDNA was much lower than population sequencing due to lower specificity [32,33]. Therefore, it is unlikely that NGS would have resulted in improved tropism accuracy for the T2 pvDNA samples. The geno2pheno tropism algorithm that has become the standard tropism prediction tool for genotypic tropism tests also has limitations. A study of plasma viral variants obtained from the "Berlin Patient", the first patient to be cured of HIV through allogenic transplantation, detected a minority non-R 5 viral population by ultradeep sequencing and geno2pheno analysis. This population however, was unable to rebound after transplantation,

Table 2 Concordance Between Plasma RNA and pvDNA Coreceptor Tropism

\begin{tabular}{llllllll}
\hline Test Method & Reference Method & non-R5 (\%) Test Ref. & Concord. (\%) & Sens. (\%) & Spec. (\%) $^{\mathbf{2}}$ & Kappa $^{\mathbf{3}}$ \\
\hline pvDNA(T1) & Plasma RNA(T1) & $33 \%$ & $24 \%$ & $88 \%$ & $92 \%$ & $86 \%$ & 0.70 \\
pvDNA(T2) & Plasma RNA(T1) & $37 \%$ & $24 \%$ & $80 \%$ & $83 \%$ & $78 \%$ & 0.53 \\
\hline
\end{tabular}

${ }^{1}$ Sensitivity is defined as concordant non-R5s between the test and reference method/reference method non-R5s.

${ }^{2}$ Specificity is defined as concordant R5s between the test and reference methods/reference method R5s.

${ }^{3}$ Kappa: a measure of inter-rater agreement; 0.41-0.60: moderate agreement; 0.61-0.80: good agreement. 
demonstrating an R5 coreceptor dependency [34]. In spite of these limitations, proviral DNA tropism determinations have proven to be predictive of virologic response in both retrospective studies of viremic patients and more recently prospectively, when used to assign virologically suppressed patients to a maraviroc-based or alternative regimen [33,35].

\section{Conclusions}

In summary, we studied HIV-1 tropism in infected women and found good concordance between plasma RNA and pvDNA coreceptor tropism results which persisted over time: $80 \%$ of women had a pvDNA tropism result that was concordant with the baseline plasma finding after a median of 4.1 years and at least a year of viral suppression. Detection of non-R5 virus was slightly more frequent in pvDNA than plasma RNA, as has been observed previously $[16,20,33]$. This finding suggests that a pvDNA tropism test may exclude slightly more individuals from treatment with a CCR5 inhibitor. However, virtually all women with detectable non-R5 virus in plasma had detectable non-R5 virus in the contemporaneous pvDNA sample, suggesting that pvDNA testing may be a promising screening option for individuals with HIV-1 suppression. $63 \%$ of the women in this study had a pvDNA tropism result of R5 at the time of virologic suppression and would have been eligible to switch to a maravirocbased regimen. Genotypic tropism determination from pvDNA is commercially available at a significantly lower cost than phenotypic tropism testing and may thus allow coreceptor tropism assessment to be extended in a cost effective manner to persons who are taking effective ART but who are being considered for CCR5 antagonist therapy. This includes patients who are experiencing adverse effects from their current antiretroviral regimen and patients receiving complex regimens who may benefit from regimen simplification. This assay may also have value as a research tool for examination of proviral HIV DNA in PBMCs.

\section{Methods}

\section{Patient Selection}

Laboratory testing of WIHS participants is performed during biannual visits and includes lymphocyte subset counts, plasma HIV-1 RNA levels and other tests as described [24]. We randomly selected a cohort of 50 paired samples collected between 1994 and 2006 from WIHS participants in the Bronx, NY and Washington, DC study sites, meeting the following criteria: a plasma viral load $\geq 5,000 \mathrm{cp} / \mathrm{mL}$ at timepoint 1 (T1), a subsequently undetectable plasma viral load of $<80$ copies $/ \mathrm{mL}$ that had been maintained for $\geq 1$ year, along with a CD4+ T-cell count of $>200$ cells $/ \mu \mathrm{L}$ at time point 2 (T2). Although most recent studies utilize a viral load cutoff of 50 copies/ $\mathrm{mL}$ to assess virologic response, a viral load cutoff of
80 copies $/ \mathrm{mL}$ was selected to be consistent with the limit of quantitation for viral load assays available at the time that these study samples were collected. Ten paired plasma and PBMC samples obtained by the WIHS from HIV-negative women were included as negative controls.

\section{Plasma HIV RNA coreceptor tropism testing}

RNA was isolated from archived plasma specimens (MagNAPure LC automated extraction system, Roche Diagnostics Corp) that were stored at $\leq-70^{\circ} \mathrm{C}$. Tropism was determined for 3 independent replicates of viral RNA, by using RT PCR and nested PCR amplification of the V3 loop region from the HIV-1 envelope gene, followed by Sanger DNA sequencing as previously described [16,29]. Tropism assignments (R5 or non-R5) were made with the geno2pheno algorithm [36] with a false positive rate (FPR) of $10 \%$ (non-R5: $\leq 10 \%$ ) the recommended cutoff when triplicate tropism determinations are employed [14].

\section{Proviral DNA coreceptor tropism testing}

Total DNA was extracted from $0.2 \mathrm{~mL}$ of cryopreserved PBMCs (MagNA Pure system, Large Volume MagNA Pure LC DNA Isolation Kit, Roche Diagnostics Corp). Three independent replicates were amplified in 2 rounds of PCR using the same primers that were used for RNA tropism testing, followed by Sanger sequencing as previously described [16,29]. Tropism was assigned using the geno2pheno algorithm [36] using the same cutoff for RNA tropism (non-R5: $\leq 10 \%$ ).

HIV-1 pvDNA quantitation was performed using a realtime quantitative PCR kit (Human HIV-DNA qPCR Detection kit, GeneMoRe, Modena, Italy) according to the manufacturer's instructions.

\section{Additional files}

Additional file 1: Figure S1. Changes in CD4+ cell counts between time points $\mathrm{T} 1$ and $\mathrm{T} 2$ (median, IQR, and range).

Additional file 2: Figure S2. pvDNA viral load assayed at $\mathrm{T} 1$ and $\mathrm{T} 2$ (mean and $95 \% \mathrm{Cl}$ [error bars]).

\section{Competing interests}

Authors REB, RMK, AAR, HBH, WAM III, and RLP are employed by Quest Diagnostics, a diagnostic testing company that offers diagnostic tests for HIV. Authors HB and BW are the inventors of six patented technologies for the determination of HIV tropism in clinical management. The patents are owned by Health Research Inc., the research foundation for the New York State Department of Health, and are licensed to Quest Diagnostics. All other authors have no competing interests.

\section{Authors' contributions}

REB and AAR conducted the experimental work. RMK performed the data analysis and participated in authoring the manuscript. WG was responsible for procuring the samples and the patient baseline characteristics. HB and BW participated in authoring the manuscript. HBH, WAM and RLP provided scientific input. KA and MY participated in the study design on behalf of WIHS. All authors read and approved the final manuscript. 


\section{Acknowledgements}

We thank the participants in the Women's Interagency HIV Study (WIHS). The study was funded by NIH grants UO1-Al-035004 and UO1-Al-034994 and by Quest Diagnostics.

Data in this manuscript were collected by the Women's Interagency HIV Study (WIHS). The contents of this publication are solely the responsibility of the authors and do not represent the official views of the National Institutes of Health (NIH). WIHS (Principal Investigators): Bronx WIHS (Kathryn Anastos), U01-Al-035004; Metropolitan Washington WIHS (Mary Young), U01-Al-034994. H.Burger and B.Weiser are faculty members in the Department of Medicine, Division of Infection Disease, UC Davis School of Medicine and Sacramento VA Medical Center, Sacramento, CA. VA resources partly supported this research.

\section{Author details}

${ }^{1}$ Focus Diagnostics, a Subsidary of Quest Diagnostics, San Juan Capistrano, CA, USA. ${ }^{2}$ University of California, Davis School of Medicine, and Sacramento VA Medical Center, Sacramento, CA, USA. ${ }^{3}$ Albert Einstein College of Medicine, and Montefiore Medical Center, Bronx, NY, USA. ${ }^{4}$ Georgetown University, Washington, DC, USA. ${ }^{5}$ Quest Diagnostics, Baltimore, MD, USA.

\section{Received: 2 December 2014 Accepted: 12 April 2015}

\section{Published online: 18 April 2015}

\section{References}

1. Berger EA, Murphy PM, Farber JM. Chemokine receptors as HIV-1 coreceptors: roles in viral entry, tropism, and disease. Annu Rev Immunol. 1999;17:657-700.

2. Goodenow MM, Collman RG. HIV-1 coreceptor preference is distinct from target cell tropism: a dual-parameter nomenclature to define viral phenotypes. J Leukoc Biol. 2006;80:965-72.

3. Ray N, Doms RW. HIV-1 coreceptors and their inhibitors. Curr Top Microbiol Immunol. 2006;303:97-120.

4. Brumme ZL, Goodrich J, Mayer HB, Brumme CJ, Henrick BM, Wynhoven B, et al. Molecular and clinical epidemiology of CXCR4-using HIV-1 in a large population of antiretroviral-naive individuals. J Infect Dis. 2005;192:466-74.

5. Chalmet K, Dauwe K, Foquet L, Baatz F, Seguin-Devaux C, Van Der Gucht B, et al. Presence of CXCR4-using HIV-1 in patients with recently diagnosed infection: correlates and evidence for transmission. J Infect Dis. 2012;205:174-84.

6. Connor RI, Sheridan KE, Ceradini D, Choe S, Landau NR. Change in coreceptor use correlates with disease progression in HIV-1-infected individuals. J Exp Med. 1997;185:621-8.

7. Irlbeck DM, Amrine-Madsen H, Kitrinos KM, Labranche CC, Demarest JF. Chemokine (C-C motif) receptor 5-using envelopes predominate in dual/ mixed-tropic HIV from the plasma of drug-naive individuals. Aids. 2008;22:1425-31.

8. Moyle GJ, Wildfire A, Mandalia S, Mayer H, Goodrich J, Whitcomb J, et al. Epidemiology and predictive factors for chemokine receptor use in HIVinfection. J Infect Dis. 2005;191:866-72.

9. Poveda E, Briz V, de Mendoza C, Benito JM, Corral A, Zahonero N, et al. Prevalence of X4 tropic HIV-1 variants in patients with differences in disease stage and exposure to antiretroviral therapy. J Med Virol. 2007;79:1040-6.

10. Shankarappa R, Margolick JB, Gange SJ, Rodrigo AG, Upchurch D, Farzadegan $\mathrm{H}$, et al. Consistent viral evolutionary changes associated with the progression of human immunodeficiency virus type 1 infection. J Virol. 1999;73:10489-502.

11. Daar ES, Kesler KL, Petropoulos CJ, Huang W, Bates M, Lail AE, et al. Baseline HIV type 1 coreceptor tropism predicts disease progression. Clin Infect Dis. 2007:45:643-9.

12. Weiser B, Philpott S, Klimkait T, Burger H, Kitchen C, Burgisser P, et al. HIV-1 coreceptor usage and CXCR4-specific viral load predict clinical disease progression during combination antiretroviral therapy. AIDS. 2008;22:469-79.

13. Dorr P, Westby M, Dobbs S, Griffin P, Irvine B, Macartney M, et al. Maraviroc (UK-427,857), a potent, orally bioavailable, and selective small-molecule inhibitor of chemokine receptor CCR5 with broad-spectrum anti-human immunodeficiency virus type 1 activity. Antimicrob Agents Chemother. 2005:49:4721-32.

14. Vandekerckhove LP, Wensing AM, Kaiser R, Brun-Vezinet F, Clotet B, De Luca A, et al. European guidelines on the clinical management of HIV-1 tropism testing. Lancet Infect Dis. 2011;11:394-407.

15. Svicher V, Alteri C, Montano M, Nori A, D'Arrigo R, Andreoni M, et al. Genotypic testing on HIV-1 DNA as a tool to assess HIV-1 co-receptor usage in clinical practice: results from the DIVA study group. Infection. 2014;42:61-71.
16. Brown J, Burger H, Weiser B, Pollard RB, Li XD, Clancy L, et al. A genotypic HIV-1 proviral DNA coreceptor tropism assay: characterization in viremic subjects. AIDS Res Ther. 2014;11:14.

17. Paar C, Geit M, Stekel H, Berg J. Genotypic prediction of human immunodeficiency virus type 1 tropism by use of plasma and peripheral blood mononuclear cells in the routine clinical laboratory. J Clin Microbiol. 2011:49:2697-9.

18. Parisi SG, Andreoni C, Sarmati L, Boldrin C, Buonomini AR, Andreis S, et al. HIV coreceptor tropism in paired plasma, peripheral blood mononuclear cell, and cerebrospinal fluid isolates from antiretroviral-naive subjects. J Clin Microbiol. 2011;49:1441-5.

19. Svicher V, Alteri C, Montano M, D'Arrigo R, Andreoni M, Angarano G, et al. Performance of genotypic tropism testing on proviral DNA in clinical practice: results from the DIVA study group. New Microbiol. 2012;35:17-25.

20. Verhofstede C, Brudney D, Reynaerts J, Vaira D, Fransen K, De Bel A, et al. Concordance between HIV-1 genotypic coreceptor tropism predictions based on plasma RNA and proviral DNA. HIV Med. 2011;12:544-52.

21. Meini G, Rossetti B, Bianco C, Ceccherini-Silberstein F, Di Giambenedetto S, Sighinolfi $\mathrm{L}$, et al. Longitudinal analysis of HIV-1 coreceptor tropism by single and triplicate HIV-1 RNA and DNA sequencing in patients undergoing successful first-line antiretroviral therapy. J Antimicrob Chemother. 2014;69:735-41.

22. Seclen E, Del Mar GM, De Mendoza C, Soriano V, Poveda E. Dynamics of HIV tropism under suppressive antiretroviral therapy: implications for tropism testing in subjects with undetectable viraemia. J Antimicrob Chemother. 2010;65:1493-6.

23. Soulie C, Fourati S, Lambert-Niclot S, Malet I, Wirden M, Tubiana R, et al. Factors associated with proviral DNA HIV-1 tropism in antiretroviral therapytreated patients with fully suppressed plasma HIV viral load: implications for the clinical use of CCR5 antagonists. J Antimicrob Chemother. 2010;65:749-51.

24. Bacon MC, von Wyl V, Alden C, Sharp G, Robison E, Hessol N, et al. The Women's Interagency HIV Study: an observational cohort brings clinical sciences to the bench. Clin Diagn Lab Immunol. 2005;12:1013-9.

25. Kearney MF, Spindler J, Shao W, Yu S, Anderson EM, O'Shea A, et al. Lack of detectable HIV-1 molecular evolution during suppressive antiretroviral therapy. PLoS Pathog. 2014;10, e1004010.

26. Fletcher CV, Staskus K, Wietgrefe SW, Rothenberger M, Reilly C, Chipman JG, et al. Persistent HIV-1 replication is associated with lower antiretroviral drug concentrations in lymphatic tissues. Proc Natl Acad Sci U S A. 2014;111:2307-12.

27. Maldarelli F, Wu X, Su L, Simonetti FR, Shao W, Hill S, et al. HIV latency. Specific HIV integration sites are linked to clonal expansion and persistence of infected cells. Science. 2014;345:179-83.

28. Soulie C, Lambert-Niclot S, Wirden M, Simon A, Valantin MA, Fourati S, et al. Low frequency of HIV-1 tropism evolution in patients successfully treated for at least 2 years. AIDS. 2011;25:537-9.

29. Kagan RM, Johnson EP, Siaw M, Biswas P, Chapman DS, Su Z, et al. A genotypic test for HIV-1 tropism combining Sanger sequencing with ultradeep sequencing predicts virologic response in treatment-experienced patients. PLoS One. 2012;7, e46334.

30. Swenson LC, Daumer M, Paredes R. Next-generation sequencing to assess HIV tropism. Curr Opin HIV AIDS. 2012;7:478-85.

31. Swenson LC, Mo T, Dong WW, Zhong X, Woods CK, Thielen A, et al. Deep V3 sequencing for HIV type 1 tropism in treatment-naive patients: a reanalysis of the MERTT trial of maraviroc. Clin Infect Dis. 2011;53:732-42.

32. Pou C, Codoner FM, Thielen A, Bellido R, Perez-Alvarez S, Cabrera C, et al. HIV-1 tropism testing in subjects achieving undetectable HIV-1 RNA: diagnostic accuracy, viral evolution and compartmentalization. PLoS One. 2013;8, e67085.

33. Swenson LC, Dong WW, Mo T, Demarest J, Chapman D, Ellery S, Heera J, Valdez H, Poon AF, Harrigan PR: Use of Cellular HIV DNA to Predict Virologic Response to Maraviroc: Performance of Population-based and Deep Sequencing. Clin Infect Dis. 2013;56:1659-1666.

34. Symons J, Vandekerckhove L, Hutter G, Wensing AM, van Ham PM, Deeks SG, et al. Dependence on the CCR5 coreceptor for viral replication explains the lack of rebound of CXCR4-predicted HIV variants in the Berlin patient. Clin Infect Dis. 2014;59:596-600.

35. Garcia F, Poveda E, Perez-Elias MJ, Quero JH, Ribas MA, Martinez-Madrid OJ, et al. Genotypic tropism testing in proviral DNA to guide maraviroc initiation in aviremic subjects: 48-week analysis of the PROTEST study. J Int AIDS Soc. 2014;17:19520.

36. Lengauer T, Sander O, Sierra S, Thielen A, Kaiser R. Bioinformatics prediction of HIV coreceptor usage. Nat Biotechnol. 2007;25:1407-10. 